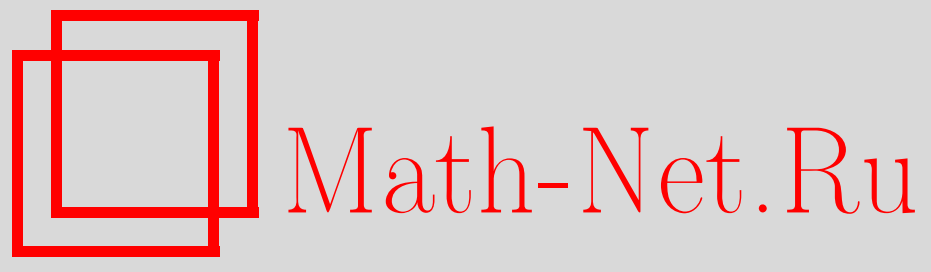

В. Д. Володин, Геометрическая реализация $\gamma$-векторов 2-усеченных кубов, УМН, 2012, том 67, выпуск 3, 181182

DOI: https://doi.org/10.4213/rm9482

Использование Общероссийского математического портала Math-Net.Ru подразумевает, что вы прочитали и согласны с пользовательским соглашением http://www . mathnet.ru/rus/agreement

Параметры загрузки:

IP : 54.197 .130 .99

26 апреля 2023 г., 14:55:20

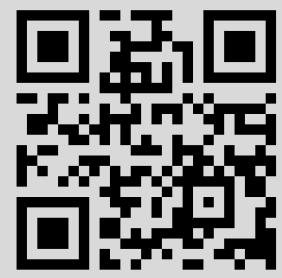




\section{Геометрическая реализация $\gamma$-векторов 2-усеченных кубов}

\section{В. Д. Володин}

Пусть $f_{i}$ - количество $i$-мерных граней простого $n$-многогранника $P$. Набор чисел $\left(f_{0}, \ldots, f_{n}\right)$ называется его $f$-вектором. Полином $F(P)(\alpha, t)=\alpha^{n}+f_{n-1} \alpha^{n-1} t+\cdots+$ $f_{1} \alpha t^{n-1}+f_{0} t^{n}$ называется $F$-полиномом, а полином $H(P)(\alpha, t)=h_{0} \alpha^{n}+h_{1} \alpha^{n-1} t+$ $\cdots+h_{n-1} \alpha t^{n-1}+h_{n} t^{n}=F(P)(\alpha-t, t)$ называется $H$-полиномом.

Соотношения Дена-Соммервилля (см. [9]) эквивалентны симметричности $H(P)$ для простых многогранников, следовательно, $H(P)$ может быть представлен в виде

$$
H(P)=\sum_{i=0}^{[n / 2]} \gamma_{i}(\alpha t)^{i}(\alpha+t)^{n-2 i}
$$

Набор коэффициентов $\left(\gamma_{0}, \gamma_{1}, \ldots, \gamma_{[n / 2]}\right)$ называется $\gamma$-вектором. Полином $\gamma(P)(\tau)=$ $\gamma_{0}+\gamma_{1} \tau+\cdots+\gamma_{[n / 2]} \tau^{[n / 2]}$ называется $\gamma$-полиномом.

Симплициальный комплекс называется флаговым, если любой набор его попарно инцидентных вершин образует симплекс. Простой многогранник называется флаговым, если граница его двойственного многогранника является флаговым симплициальным комплексом, т. е. любой набор его попарно пересекающихся граней имеет непустое пересечение. Гипотеза Гала (см. [6]) в случае выпуклых многогранников утверждает, что простой флаговый многогранник имеет неотрицательный $\gamma$-вектор.

ОПРедЕЛЕниЕ 1. Простой многогранник $P^{n}$ называется 2-усеченным кубом, если он может быть получен из куба $I^{n}$ последовательностью срезок граней коразмерности 2 (граница двойственного многогранника получается из кросс-политопа последовательностью звездных подразделений вдоль ребер).

Класс 2-усеченных кубов введен в работе [2], одним из результатов которой является доказательство гипотезы Гала для этого класса. В [3] было показано, что известные классы простых многогранников (граф-ассоциэдры, граф-кубиэдры, флаговые нестоэдры) являются 2-усеченными кубами. Классу флаговых нестоэдров принадлежат обобщенные ассоциэдры серии $A$ (многогранники Сташефа) и серии $B$ (многогранники Ботта-Таубса). В [7] показано, что обобщенные ассоциэдры серии $D$ не являются флаговыми нестоэдрами, но тем не менее являются 2-усеченными кубами. В [1] для каждого флагового нестоэдра $P$ построен флаговый симплициальный комплекс $\Delta_{P}$ такой, что $\gamma(P)=f\left(\Delta_{P}\right)$. Ранее такой комплекс был построен для многогранников Сташефа, многогранников Ботта-Таубса и пермутоэдров (см. [8]). Центральным результатом настоящей работы является следующая теорема.

Теорема 2. Для любого 2-усеченного куба $P^{n}$ существует флаговый симплициальный комплекс $\Delta_{P}$ такой, что $\gamma(P)=f\left(\Delta_{P}\right)$. Здесъ $f(K)(t)=1+f_{0} t+f_{1} t^{2}+\cdots+$ $f_{d} t^{d+1}$, где $d$ - размерность комплекса $K$.

Доказательство. Рассмотрим 2-усеченный куб $P^{n}$ и множество $W(P)$ всех его гиперграней, полученных в результате последовательности срезок граней. Для каждой грани $G \subset P$, в том числе и для $G=P$, построим флаговый симплициальный комплекс $\Delta_{G}$ на множестве вершин $W(P)$ такой, что $f\left(\Delta_{G}\right)(t)=\gamma(G)(t)$ и если $G_{1} \subset G_{2}$, то $\Delta_{G_{1}} \subset \Delta_{G_{2}}$.

Пусть $P=I^{n}$, тогда $W(P)=\varnothing$. В этом случае положим $\Delta_{G}=\varnothing$ для всех граней.

Работа выполнена при поддержке гранта Правительства РФ по постановлению № 220, договор № 11.G34.31.0053. 
Допустим, что для многогранника $P$ уже построено семейство требуемых симплициальных комплексов, а многогранник $P^{\prime}$ получен из $P$ срезкой грани $G_{0}$ коразмерности 2. Тогда $W\left(P^{\prime}\right)=W(P) \cup\left\{v\left(G_{0}\right)\right\}$, где вершина $v\left(G_{0}\right)$ соответствует новой гиперграни многогранника $P^{\prime}$. Рассмотрим произвольную грань $G^{\prime} \subset P^{\prime}$. Если грань $G^{\prime}$ получена из некоторой грани $G$ срезкой ее грани $F$ коразмерности 2 , то положим $\Delta_{G^{\prime}}=\Delta_{G} \cup$ Cone $\left(\Delta_{F}\right)$. $f$-полиномы комплексов $\Delta_{G^{\prime}}$ и $\Delta_{G}$ связаны соотношением

$$
f\left(\Delta_{G^{\prime}}\right)=f\left(\Delta_{G}\right)+t f\left(\Delta_{F}\right) .
$$

Аналогичным соотношением (см. [2]) связаны $\gamma$-векторы граней $G$ и $G^{\prime}$ :

$$
\gamma\left(G^{\prime}\right)=\gamma(G)+\tau \gamma(F)
$$

Следовательно, $f\left(\Delta_{G^{\prime}}\right)=\gamma\left(G^{\prime}\right)$. Если грань $G^{\prime}$ получена из некоторой грани $G$ срезкой грани коразмерности 1 или $G^{\prime}$ совпадает с $G$, то положим $\Delta_{G^{\prime}}=\Delta_{G}$.

Очевидно, что $f\left(\Delta_{G^{\prime}}\right)(t)=\gamma\left(G^{\prime}\right)(t)$ и если $G_{1}^{\prime} \subset G_{2}^{\prime}$, то $\Delta_{G_{1}^{\prime}} \subset \Delta_{G_{2}^{\prime}}$.

Комплекс $\Delta_{P^{\prime}}$ является флаговым - как объединение флаговых комплесов $\Delta_{G^{\prime}}$ и Cone $\left(\Delta_{F}\right)$, пересечение которых есть также флаговый комплекс $\Delta_{F}$.

Обратим внимание, что комплекс $\Delta_{P}$ зависит от выбранной последовательности срезок.

Обозначим через $\left(\begin{array}{l}n \\ k\end{array}\right)_{r}$ число $k$-клик в графе Турана $T_{n, r}$. Для натуральных чисел $m, k$ и $r \geqslant k$ однозначно определено разложение $m=\left(\begin{array}{c}n_{k} \\ k\end{array}\right)_{r}+\cdots+\left(\begin{array}{l}n_{k-s} \\ k-s\end{array}\right)_{r-s}$, где $n_{k-i}-\left[\frac{n_{k-i}}{r-i}\right]>n_{k-i-1}$ для всех $0 \leqslant i<s$ и $n_{k-s} \geqslant k-s>0$. Положим $m^{\langle k\rangle_{r}}=\left(\begin{array}{c}n_{k} \\ k+1\end{array}\right)_{r}+\cdots+\left(\begin{array}{c}n_{k-s} \\ k-s+1\end{array}\right)_{r-s}$.

Согласно [5], $f$-вектор флагового симплициального комплекса является $f$-вектором некоторого сбалансированного симплициального комплекса. Отсюда, используя неравенства Франкла-Фюреди-Калаи (см. [4]) для $f$-векторов симплициальных комплексов, получаем результат, который в случае флаговых нестоэдров содержится в [1].

СледСтвие 3. Пусть $P^{n}$ есть 2-усеченный куб. Тогда: 1) $\gamma_{0}=1$; 2) $0 \leqslant \gamma_{i+1} \leqslant$ $\gamma_{i}^{\langle i\rangle_{r}}$, əде $r=[n / 2]$.

\section{Список литературы}

[1] N. Aisbett, Frankl-Füredi-Kalai inequalities on the $\gamma$-vectors of flag nestohedra, arXiv: 1203.4715. [2] В. М. Бухштабер, В.Д. Володин, Изв. РАН. Сер. матем., 75:6 (2011), 17-46; англ. пер.: V. M. Buchstaber, V.D. Volodin, Izv. Math., 75:6 (2011), 1107-1133. [3] V. Buchstaber, V. Volodin, "Combinatorial 2-truncated cubes and applications", Associahedra, Tamari lattices and related structures. Tamari Memorial Festschrift, Progr. Math., 299, eds. F. Müller-Hoissen, J. Pallo, J. Stasheff, Birkhäuser, Boston, MA, 2012, 161-186. [4] P. Frankl, Z. Füredi, G. Kalai, Math. Scand., 63:2 (1988), 169-178. [5] A. Frohmader, Face vectors of flag complexes, arXiv: math/0605673. [6] Ś. R. Gal, Discrete Comput. Geom., 34:2 (2005), 269-284; arXiv: math/0501046. [7] М. А. Горский, УМH, 65:6(396) (2010), 185-186; англ. пер.: M. A. Gorsky, Russian Math. Surveys, 65:6 (2010), 1178-1180. [8] E. Nevo, T. K. Peterson, Discrete Comput. Geom., 45:3 (2011), 503-521. [9] G. M. Ziegler, Lectures on polytopes, Grad. Texts in Math., 152, Springer-Verlag, New York, 1995, x+370 pp.

В. Д. Володин (V.D. Volodin)

Математический институт им. В. А. Стеклова РАН; Лаборатория дискретной и вычислительной геометрии им. Б. Н. Делоне, Ярославль

E-mail: volodinvadim@gmail.com
Представлено В. М. Бухштабером Принято редколлегией 10.04.2012 